\title{
Statement on continuing care for older adults with psychiatric disorder
}

A consensus meeting attended by members of the College together with representatives from Age Concern, the Alzheimer's Disease Society, the Association of Directors of Social Services, the British Geriatrics Society, the Department of Health and the Royal College of Physicians was held at the College in March 1995.

The following statement was subsequently agreed by the Section for the Psychiatry of Old Age, and was approved by the Council of the College on 28 April 1997.

Consultant-led multi-disciplinary community teams in old age psychiatry linked to appropriate hospital resources should be available as part of a spectrum of services which also involves family and friends, voluntary agencies, general practitioners, social services, other NHS providers and the independent sector. Their specific role is to assist in the assessment and management of older patients with dementia and other mental illness in a way that aims to identify and meet the needs of individual patients and their carers.

Despite full assessment and support at home and in other settings a small proportion of patients will require continuing care in NHS units staffed by psychiatric nurses and other disciplines under the care of consultants in old age psychiatry. Characteristics of patients likely to need admission to such units include the following:

(a) sustained or frequently recurrent difficult behaviour including aggression and violence, sexual disinhibition, intractable noisiness, interfering with other residents, persistent absconding and violent resistance to necessary care which arises from dementia or other serious psychiatric disorder and is of a severity that cannot be managed in other settings except by inappropriate use of sedation;

(b) physical illness and sensory problems if they are associated with problems listed above and the needs of the patient cannot be better met in another setting (e.g. NHS medical ward, hospice for terminal care);

(c) dementia or other serious psychiatric disorder and failure to cope or more rapid deterioration in other care settings.

Eligibility criteria for NHS continuing care should be framed to include patients with these characteristics. The vast majority of patients will be 'informal' but sometimes detention under the Mental Health Act may be justified.

NHS units should provide a model of quality care. The accommodation, skills and level of staffing should take account of the needs of patients in order to maximise residual ability and provide the best possible quality of life. Good units will usually include no more than 20 beds, mostly or all in single rooms, with an adequate choice of a variety of day spaces and a protected garden. They should be local to the patient's home to facilitate visiting and should offer respite care. They will also often offer day places and serve as a base for outreach into the local community for the local psychiatric consultant and community team for older adults.

The extent, reasons for, and impact of the current wide local variations in NHS provision should be investigated. Regularly updated local registers of vulnerable patients with dementia based on general practice computerised records would help ensure needs are recognised and met. Their feasibility should be explored.

Approved by Council, 28 April 1997 